\title{
CORRECTION
}

\section{Correction Re: Tissue Engineering, Part A, 2016;22(1-2):17-30}

In the January 2016 issue of Tissue Engineering, Part A (vol. 22, no. 1-2;17-30), in the article entitled, "Assessment of Methods for Rapid Intraoperative Concentration and Selection of Marrow-Derived Connective Tissue Progenitors for Bone Regeneration Using the Canine Femoral Multidefect Model" by Luangphakdy V et al., a correction was received too late to be inserted in the article before the issue was printed.

The legend for Figure 4 referred to Supplementary Figure 7 in error. The legend should have said "as illustrated in Figure 1."

The corrected legend is reprinted here in full, with the corrected wording in bold:

FIG. 4. Mean percent bone volume (\%BV) plots versus the radial position in the defect. PC: The PC region represents the region between 8 and $12 \mathrm{~mm}$ from the bottom of the grafted site, which is adjacent to the cortex. IM: The IM region is defined as the region between 3 and $7 \mathrm{~mm}$ from the bottom of the grafted site, which is separated by several millimeters from the endosteal source of osteogenic cells and bound only by cells from the marrow cavity, as illustrated in Figure 1. The highest $\% \mathrm{BV}$ was found near the periphery of the defect, with generally lower $\% \mathrm{BV}$ in the middle and central regions. Mean $\% \mathrm{BV}$ was highest in the PC region of the defect and lower in the IM region $(\mathrm{p}<0.001)$. The graphs in the right column provide a secondary comparison of the DS processing methods used in Cohort I and II, showing. There was no statistical difference between DS samples processed without coagulation, and DS-T samples processed with calcium thrombin was added to the DS concentrate.

The authors sincerely regret this error. 